\title{
Utilización de los grupos clínicos ajustados en un centro de atención primaria español: estudio retrospectivo de base poblacional
}

\author{
Antoni Sicras-Mainar, ${ }^{1}$ Olga Pané-Mena, ${ }^{2}$ Teresa Martínez-Ruíz, ${ }^{2}$ \\ Josep Lluís Fernández-Roure, ${ }^{2}$ Jordi Prats-Sánchez, ${ }^{2}$ Lourdes Santafé, ${ }^{3}$ \\ Ruth Navarro-Artieda, ${ }^{1}$ Alejandra Prados-Torres ${ }^{4}$ y José Estelrich-Bennasar ${ }^{5}$
}

Forma de citar Sicras-Mainar A, Pané-Mena O, Martínez-Ruíz T, Fernández-Roure JL, Prats-Sánchez J, Santafé L, et al. Utilización de los grupos clínicos ajustados en un centro de atención primaria español: estudio retrospectivo de base poblacional. Rev Panam Salud Publica. 2010;27(1):49-55.

RESUMEN Objetivos. Evaluar los resultados de la aplicación del sistema de clasificación mediante grupos clínicos ajustados (GCA) en un centro de atención primaria de salud (APS) de una población española.

Métodos. Estudio transversal retrospectivo a partir de los registros médicos informatizados de los pacientes atendidos ambulatoriamente durante 2007 en el centro de salud La Roca, administrado por una empresa de gestión de servicios de salud en La Roca del Vallès, Barcelona, España. Los GCA se conformaron según la Clasificación Internacional de Enfermedades, 9. ${ }^{a}$ revisión, modificación clínica. Se calcularon los pesos relativos medios en dólares estadounidenses de cada GCA respecto al costo medio total $y$, a partir de ellos, se crearon las bandas de utilización de recursos. Se determinaron los indices de riesgo (IR) y eficiencia (IE) con respecto a 2006 y se estimó el poder explicativo de la clasificación empleada.

Resultados. Se estudiaron 8294 pacientes, para una cobertura de 82,7\%, con una media de 4,1 episodios por paciente, 6,9 visitas por paciente y 5,7 visitas por habitante al año. A siete GCA correspondió $51,0 \%$ de los pacientes atendidos. El IR fue de 1,015, el IE en las visitas de 0,975 y el poder explicativo de la clasificación en GCA fue de 53,4\% para las visitas y de 74,8\% para los episodios.

Conclusiones. El sistema de GCA permitió agrupar a los pacientes de una población según su estado clínico y puede ayudar, entre otros aspectos, en la asignación de recursos y la evaluación de la eficiencia de los equipos de APS.

Palabras clave Grupos diagnósticos relacionados; atención primaria de salud; eficiencia organizacional; España.

Dirección de Planificación, Badalona Serveis Assistencials, S.A., Badalona, Barcelona, España. La correspondencia se debe dirigir a Antoni Sicras Mainar, Badalona Serveis Assistencials, S.A., Calle Gaietà Soler No. 6-8 entresuelo, Badalona CP 08911, Barcelona, España. Correo electrónico: asicras@bsa.cat

2 Dirección de Proyectos Sanitarios y Sociales, Consorci Hospitalari de Catalunya, Barcelona, España. Área Básica de Salud, La Roca del Vallès, Barcelona, España.

4 Investigación en Salud, Instituto Aragonés de Ciencias de la Salud, Zaragoza, España.
La implantación de un sistema de información sanitario en el ámbito de la atención primaria de salud (APS) debe responder a la necesidad de establecer un instrumento de evaluación del estado de salud de la población y de las activi-

5 Dirección de Planificación, Gerencia de Atención Primaria, Mallorca, España. dades que se realizan sobre ella. La mayoría de los sistemas de clasificación de pacientes se han diseñado en los Estados Unidos de América con la finalidad de disponer de una herramienta que facilite medir la utilización de los recursos hospitalarios. De ellos, los únicos que presentan un elevado poder explicativo sobre los costos de la asistencia durante 
la hospitalización convencional son los basados en los grupos relacionados con el diagnóstico (1-3).

Los sistemas de clasificación de pacientes no se han empleado de forma generalizada en los servicios de atención ambulatoria, en particular en los de APS, y de ellos, los que agrupan a los pacientes según su consumo de recursos pueden facilitar la clasificación de los usuarios de los servicios de salud. De esos sistemas, en el conocido como grupos clínicos ajustados (GCA), desarrollado por Starfield (4) y Weiner (5), se clasifican los pacientes según las enfermedades que presentan durante un período de tiempo, lo que permite medir el "grado de enfermedad" en la población a partir de los niveles de morbilidad y comorbilidad.

Actualmente existen experiencias de gestión que promueven la separación de la financiación, la compra y la provisión de servicios, pero se necesitan instrumentos más precisos de evaluación y medición de la actividad asistencial realizada. En los últimos años se observa una tendencia a utilizar la financiación per cápita como mecanismo para asignar los recursos asistenciales. Algunos sistemas de salud caracterizados por la competencia entre las aseguradoras - como los de Alemania y los Estados Unidos- se fundamentan en el riesgo en padecer la enfermedad, mientras que otros — como los del Reino Unido y Suecia- utilizan este enfoque para redistribuir los recursos más equitativamente (6).

En España, la capacidad de los sistemas de clasificación de pacientes como método de medición para responder a las necesidades de la gestión de los servicios de salud en la APS es incierta. En este sentido, ni siquiera se dispone de un conjunto mínimo básico normalizado de datos y, al parecer, hay dudas sobre los propios instrumentos y su posible ajuste a un ámbito sanitario con características diferentes de aquel para el que se diseñaron (7). En cuanto a la aplicación de los GCA en España, aunque hay algunos estudios generales, aún son pocas las experiencias de aplicación práctica que respalden la consistencia de los resultados (8-12).

Los objetivos del presente estudio fueron evaluar los resultados de la aplicación del sistema de GCA en un centro de APS de una población española, determinar los índices de riesgo y eficiencia y estimar el poder explicativo de la clasificación empleada.

\section{MATERIALES Y MÉTODOS}

Se efectuó un estudio transversal retrospectivo a partir de los registros médicos informatizados de los pacientes que recibieron atención ambulatoria de APS en el centro de salud La Roca, administrado por la empresa de gestión de servicios de salud PROSS (Proyectos Sanitarios y Sociales, S.A.) en el municipio La Roca del Vallès, provincia de Barcelona, en la comunidad autónoma de Cataluña, España.

En este municipio viven 10035 habitantes, de ellos 17,8\% son menores de 14 años (pacientes pediátricos) y $15,6 \%$ son mayores de 64 años. La mayoría de la población de este municipio es urbana o semiurbana, de nivel socioeconómico medio o bajo, dedicados fundamentalmente a labores industriales y agrícolas. Los servicios de salud, tanto públicos como privados (concertados con el Servicio Catalán de la Salud), funcionan mediante un modelo de gestión descentralizado.

En el estudio participaron todos los pacientes atendidos entre enero $\mathrm{y}$ diciembre de 2007, excepto los trasladados a otros centros de salud. Como grupo de referencia se tomó la población atendida durante 2006, con el mismo criterio de exclusión, ya que hasta el momento no existe una "norma" para el país por la falta de un conjunto mínimo básico normalizado de datos.

\section{Variables y agrupación de pacientes}

Las variables independientes fueron la edad y el sexo y las dependientes fueron las visitas realizadas y los episodios atendidos. Se utilizó la variable edad para agrupar a los pacientes en pediátricos (de 0 a 14 años), atendidos en la unidad básica de asistencia (UBA) No. 1, y de medicina familiar (mayores de 14 años), atendidos en las UBA 2-7. Cada UBA está constituida por el conjunto de pacientes adscritos a un profesional médico. Las visitas se definieron como el contacto entre un miembro del equipo de salud (médicos, enfermeras, odontólogos o trabajadores sociales) y el paciente por una misma demanda o problema de salud, ya fuera en el propio centro o en el domicilio. Cuando en la visita participaron dos profesionales, se contabilizó por el que figuraba en la agenda de citaciones. Se excluyeron del estudio las visitas atendidas por médicos especialistas hospitalarios en sus centros.
La intensidad de uso se calculó como el porcentaje de pacientes atendidos con respecto a la población total que corresponde a esos servicios. Se consideraron hiperfrecuentadores a los pacientes que realizaron más de 20 visitas en un año.

Un episodio se definió como un ciclo de atención por una enfermedad o una demanda explícita realizada por el paciente que requiere uno o varios contactos con miembros del equipo de salud. A cada episodio le correspondió un diagnóstico o motivo de consulta, agrupados según la segunda versión de la Clasificación Internacional de la Atención Primaria en la Comunidad Europea (CIAP-2) (13). Los episodios no repetidos se contabilizaron por las fechas de registro en las historias clínicas, según el motivo de la consulta, ya fuera un trastorno agudo o crónico, independientemente de la fecha del diagnóstico.

Mediante el programa Grouper $\mathrm{ACG}^{\circledR}$ versión 7.0 (14) se establecieron 106 GCA, excluyentes entre sí, a partir de la edad, el sexo y el diagnóstico o motivo de consulta. Para ello se constituyó un grupo de trabajo formado por cinco profesionales (un documentalista, dos médicos clínicos y dos técnicos consultores) que se encargó de convertir la CIAP-2 a la Clasificación Internacional de Enfermedades, 9. ${ }^{a}$ revisión, modificación clínica (CIE-9-MC) (3). Los criterios empleados se basaron en el tipo de relación establecida entre los códigos de ambas clasificaciones (nula: de uno a ninguno; unívoca: de uno a uno; o múltiple: de uno a varios) (8).

La conformación de los GCA a partir de la CIE-9-MC se realizó en cuatro etapas, en las que se definieron los diferentes elementos del sistema de GCA (14). En la primera etapa se agruparon los diagnósticos de la CIE-9-MC en 32 grupos de diagnóstico ambulatorio; en la segunda etapa se redujeron a 12 grupos ajustados de diagnóstico ambulatorio, según la recurrencia de la enfermedad. En la tercera etapa se establecieron 25 categorías ambulatorias mayores y en la cuarta etapa - a partir de la combinación de los elementos anteriores, la edad y el sexo- se conformaron los 106 GCA. Cada paciente se asignó exclusivamente a un único GCA $(8,14)$.

El método aplicado permite calcular los pesos relativos medios (PRM) en dólares estadounidenses $(4,5)$ de cada grupo respecto al costo medio total $\mathrm{y}$, a partir de ellos, se crearon las bandas de 
utilización de recursos (BUR), que son agregaciones de GCA que presentan un consumo similar de recursos. En las BUR, los pacientes se agruparon según su morbilidad en cinco categorías excluyentes entre sí: usuario sano, morbilidad baja, morbilidad moderada, morbilidad elevada o morbilidad muy elevada.

Además, se conformaron los conglomerados ampliados de diagnóstico (CAD), que permitieron seleccionar los pacientes con una enfermedad dada e identificar episodios específicos, sin tener en cuenta la aplicación del algoritmo de asignación de un GCA. Por ejemplo, para definir el CAD de hipertensión arterial no complicada (código CAR14) se siguieron los criterios clínicos ampliamente aceptados, según los cuales hay un control óptimo de la hipertensión arterial cuando los valores de la tensión arterial sistólica y diastólica son menores de $140 \mathrm{~mm} \mathrm{Hg} \mathrm{y} 90 \mathrm{~mm} \mathrm{Hg}$, respectivamente; en pacientes diabéticos o que sufrieron un evento cardiovascular agudo, los valores aceptados son $130 \mathrm{~mm}$ $\mathrm{Hg}$ y $80 \mathrm{~mm} \mathrm{Hg}$, respectivamente (15).

Se calculó el índice de riesgo - también conocido como carga de morbilidad o índice de casos-, para relacionar la comorbilidad de los pacientes a partir de la media de las visitas esperadas en 2007 (ajustadas por la edad, el sexo y los episodios) y las registradas en 2006 (mediante el método de comparación de estandarización indirecta). El índice de eficiencia se calculó como el cociente entre las visitas registradas (realizadas) y las esperadas (mediante el método de comparación de estandarización indirecta); valores de este índice menores que 1 reflejan una mayor eficiencia con respecto al año de referencia.

\section{Análisis de los datos}

Las variables estudiadas se analizaron mediante la prueba de conformidad de Kolmogorov-Smirnov. Se realizó la transformación logarítmica de las variables dependientes (episodios y visitas) para ajustarlas a la distribución normal y se estableció el poder explicativo de la clasificación (visitas y episodios) mediante el coeficiente de determinación $\left(R^{2}\right)$, que refleja la varianza dentro del grupo con respecto a la varianza total.

Para el análisis bifactorial se aplicaron la prueba de independencia de la correlación lineal de Pearson, el análisis de la varianza y la prueba de la ji al cuadrado. Además, se realizó un análisis de regresión lineal múltiple para relacionar tres modelos: uno utilizó la edad como variable independiente, otro empleó la edad y el sexo, y otro utilizó la edad, el sexo y el promedio de episodios por paciente; la variable dependiente en todos los casos fue el PRM. Para agrupar y relacionar las medidas se utilizó el programa informático Microsoft Access, mientras que para el análisis estadístico se empleó el paquete estadístico SPSSW v. 12.0. El nivel de significación estadística fue de $P \leq 0,05$.

Los datos se digitaron en sistemas computarizados mediante sentencias
TRANSACT-SQL, con total respeto de la confidencialidad de los registros.

\section{RESULTADOS}

De los 10035 habitantes del municipio cubierto por el centro de salud, se atendió un total de 8294 pacientes, lo que representó un incremento de 7,3\% con respecto a los 7731 pacientes atendidos en 2006. En promedio, en el año se registraron 4,1 episodios y 6,9 visitas por paciente (frente a 4,1 y 6,9 en 2006, respetivamente). La edad promedio fue de 38,2 años (frente a 38,3 en 2006; $P=0,623$ ) (cuadro 1). Se observó una mayor frecuencia anual de visitas por habitante en 2007 con respecto a 2006 (5,7 frente a 5,5, respectivamente; $P=0,011$ ), superior si se toman en cuenta solamente los pacientes del servicio de pediatría $(7,0$ frente a 7,7, respectivamente; $P<0,001$ ). La intensidad de uso en 2007 fue de $82,7 \%$, superior en el servicio de pediatría $(91,9 \%)$.

Se observó una considerable heterogeneidad en el número de habitantes cubiertos por este centro de salud en general, así como en su distribución geográfica por UBA (cuadro 2). En comparación con el conjunto total de pacientes, en los hipertensos (código CAR14 de los CAD) había una mayor proporción de mujeres (60,8\% frente a $47,8 \%$, respectivamente) y estos tenían mayor edad $(68,0$ frente a 61,4 años), BUR (3,1 frente a 2,9), PRM $(2,84$ frente a 2,26$)$ y realizaron más visitas al médico $(14,3$ frente a 11,9$)(P<0,001)$.

CUADRO 1. Características de la población atendida en los servicios de pediatría y medicina familiar (adultos) del municipio La Roca del Vallès, Barcelona, España, el año estudiado (2007) y el de referencia (2006)

\begin{tabular}{|c|c|c|c|c|c|c|c|}
\hline \multirow[b]{2}{*}{ Característica } & \multicolumn{3}{|c|}{2006} & \multicolumn{3}{|c|}{2007} & \multirow[b]{2}{*}{$P^{\mathrm{a}}$} \\
\hline & Medicina & Pediatría & Total & Medicina & Pediatría & Total & \\
\hline Población cubierta & 8233 & 1602 & 9835 & 8245 & 1790 & 10035 & $N A^{b}$ \\
\hline Pacientes atendidos & 6257 & 1474 & 7731 & 6649 & 1645 & 8294 & NA \\
\hline Episodios registrados & 25302 & 6689 & 31991 & 27362 & 6948 & 34310 & NA \\
\hline Visitas realizadas & 41369 & 12320 & 53689 & 44960 & 12485 & 57445 & NA \\
\hline Edad (años) ${ }^{c}$ & $46,7 \pm 19,2$ & $6,1 \pm 4,5$ & $38,3 \pm 21,1$ & $46,1 \pm 18,6$ & $6,1 \pm 4,3$ & $38,2 \pm 23,1$ & $>0,05$ \\
\hline Proporción de mujeres (\%) & 53,9 & 49,5 & 53,2 & 53,8 & 49,8 & 53,0 & $>0,05$ \\
\hline Episodios por paciente ${ }^{c}$ & $4,0 \pm 4,5$ & $4,5 \pm 2,2$ & $4,1 \pm 2,9$ & $4,1 \pm 3,1$ & $4,2 \pm 2,9$ & $4,1 \pm 3,0$ & $>0,05$ \\
\hline Visitas por paciente ${ }^{\mathrm{C}}$ & $6,6 \pm 9,2$ & $8,4 \pm 6,2$ & $6,9 \pm 8,4$ & $6,8 \pm 8,1$ & $7,6 \pm 7,3$ & $6,9 \pm 8,0$ & $>0,05$ \\
\hline Visitas por episodio & 1,6 & 1,8 & 1,7 & 1,6 & 1,8 & 1,7 & $>0,05$ \\
\hline Intensidad de uso (\%) & 81,0 & 92,0 & 82,8 & 80,6 & 91,9 & 82,7 & $>0,05$ \\
\hline Visitas por habitante & 5,0 & 7,7 & 5,5 & 5,5 & 7,0 & 5,7 & 0,011 \\
\hline BUR por grupoc, d & $2,2 \pm 1,1$ & $1,8 \pm 0,5$ & $2,2 \pm 0,8$ & $2,3 \pm 0,9$ & $1,9 \pm 0,7$ & $2,3 \pm 0,9$ & 0,002 \\
\hline $\mathrm{PRM}^{\mathrm{c}, \mathrm{e}}$ & $1,2 \pm 1,5$ & $0,6 \pm 0,4$ & $1,2 \pm 1,5$ & $1,3 \pm 1,7$ & $0,6 \pm 0,5$ & $1,2 \pm 1,6$ & $>0,05$ \\
\hline
\end{tabular}

a Según las pruebas de la $t$ de Student y de la ji al cuadrado. Nivel de significación $P \leq 0,05$.

bNA: no aplica.

c Se ofrece la media \pm la desviación típica.

dBUR: bandas de utilización de recursos.

e PRM: pesos relativos medios en dólares estadounidenses del costo de la asistencia $(3,4)$. 
CUADRO 2. Características y complejidad clínica de los pacientes en general y con hipertensión arterial atendidos en los servicios de medicina familiar de las diferentes unidades básicas de asistencia (UBA) del municipio La Roca del Vallès, Barcelona, España, 2007ª

\begin{tabular}{|c|c|c|c|c|c|c|}
\hline Variable & UBA 2 & UBA 3 & UBA 4 & UBA 5 & UBA 6 & UBA 7 \\
\hline \multicolumn{7}{|l|}{ Características generales } \\
\hline Población cubierta & 1179 & 2014 & 2064 & 1593 & 851 & 527 \\
\hline Pacientes atendidos & 826 & 1569 & 1589 & 1158 & 636 & 380 \\
\hline Proporción de mujeres (\%) & 54,9 & 53,1 & 52,4 & 56,0 & 50,1 & 53,7 \\
\hline Edad (años) $)^{b}$ & $40,2 \pm 14,8$ & $48,4 \pm 19,1$ & $46,2 \pm 18,1$ & $46,1 \pm 18,3$ & $43,4 \pm 18,0$ & $47,6 \pm 18,0$ \\
\hline Episodios registrados & 3349 & 7217 & 6034 & 5355 & 2760 & 1678 \\
\hline Episodios por paciente ${ }^{b}$ & $4,1 \pm 3,1$ & $4,6 \pm 3,2$ & $3,8 \pm 2,8$ & $4,6 \pm 3,2$ & $4,3 \pm 3,0$ & $4,4 \pm 3,1$ \\
\hline Visitas realizadas & 5278 & 12169 & 10253 & 8429 & 3797 & 2789 \\
\hline Visitas por paciente $^{\mathrm{b}}$ & $6,4 \pm 7,7$ & $7,8 \pm 7,9$ & $6,5 \pm 8,1$ & $7,3 \pm 6,7$ & $6,0 \pm 6,1$ & $7,3 \pm 8,0$ \\
\hline Frecuentación anual (visitas/población) & 4,5 & 6,0 & 5,0 & 5,3 & 4,5 & 5,3 \\
\hline Pacientes hiperfrecuentadores ${ }^{C}(\%)$ & 3,1 & 5,2 & 3,1 & 3,2 & 2,5 & 4,2 \\
\hline Intensidad de uso (\%) & 70,1 & 77,9 & 77,0 & 72,7 & 74,7 & 72,1 \\
\hline BUR por paciente ${ }^{b, d}$ & $2,3 \pm 0,9$ & $2,5 \pm 0,9$ & $2,3 \pm 0,9$ & $2,4 \pm 0,8$ & $2,4 \pm 0,8$ & $2,4 \pm 0,9$ \\
\hline PRM por paciente ${ }^{b, e}$ & $1,319 \pm 1,8$ & $1,604 \pm 2,0$ & $1,206 \pm 1,5$ & $1,465 \pm 1,7$ & $1,302 \pm 1,7$ & $1,440 \pm 1,8$ \\
\hline Casos de hipertensión arterial $^{\dagger}$ & 53 & 239 & 170 & 153 & 67 & 52 \\
\hline Edad (años) ${ }^{b}$ & $61,4 \pm 15,0$ & $68,0 \pm 12,2$ & $67,2 \pm 11,2$ & $66,0 \pm 11,6$ & $66,3 \pm 11,7$ & $64,9 \pm 12,3$ \\
\hline Proporción de mujeres (\%) & 50,9 & 54,4 & 52,4 & 60,8 & 47,8 & 55,8 \\
\hline BUR por paciente ${ }^{b, d}$ & $3,0 \pm 0,6$ & $3,1 \pm 0,5$ & $2,9 \pm 0,5$ & $3,0 \pm 0,5$ & $3,0 \pm 0,3$ & $3,1 \pm 0,5$ \\
\hline PRM por paciente $\mathrm{b}, \mathrm{e}$ & $2,54 \pm 2,5$ & $2,84 \pm 2,7$ & $2,26 \pm 2,2$ & $2,81 \pm 2,1$ & $2,40 \pm 1,4$ & $2,71 \pm 2,5$ \\
\hline Visitas por paciente ${ }^{b}$ & $11,9 \pm 8,2$ & $13,8 \pm 11,0$ & $14,3 \pm 16,6$ & $13,4 \pm 7,8$ & $12,5 \pm 7,5$ & $13,1 \pm 9,6$ \\
\hline Pacientes en control óptimo de HTA (\%) & 68,2 & 80,5 & 62,1 & 76,8 & 80,4 & 71,8 \\
\hline
\end{tabular}

No se observó correlación lineal directa entre estas variables y el control de la hipertensión $(P>0,05)$.

Al comparar los principales CDA se observó que, tanto en 2006 como en 2007, más de la mitad de los pacientes fueron objeto de alguna actividad preventiva $(54,1 \%$ y $54,6 \%$, respectivamente), pero en 2007 más pacientes recibieron atención por enfermedades del tracto respiratorio superior $(36,4 \%)$ que en 2006 (33,9\%; $P<0,001)$ (cuadro 3). Asimismo, se observaron incrementos significativos $(P<0,001)$ en el número de pacientes que presentaron signos y síntomas inespecíficos $(26,6 \%)$, trastornos bucales $(19,4 \%)$ e infecciones del tracto respiratorio inferior $(10,4 \%)$ en el año 2007 con respecto a 2006 (cuadro 3).

El funcionamiento de los GCA en 2006 y 2007 fue bastante homogéneo. A siete GCA correspondieron 53,0\% de los pacientes atendidos en 2006 y 51,0\% de los atendidos en 2007, mientras que en 19 GCA se agruparon $81,1 \%$ y $78,6 \%$ de los casos de esos años, respectivamente. El índice de riesgo (carga de morbilidad general) obtenido a partir de la distribución de los GCA fue de 1,015, lo que refleja que los casos atendidos en 2007

CUADRO 3. Casos registrados, según los principales conglomerados diagnósticos ampliados, Municipio La Roca del Vallès, Barcelona, España, 2006 y 2007

\begin{tabular}{|c|c|c|c|c|c|c|}
\hline \multirow[b]{2}{*}{ Código ${ }^{a}$} & \multirow[b]{2}{*}{ Descripción } & \multicolumn{2}{|c|}{2006} & \multicolumn{2}{|c|}{2007} & \multirow{2}{*}{$\begin{array}{c}\text { Incremento } \\
(\%)\end{array}$} \\
\hline & & No. & $\% \mathrm{~b}$ & No. & $\% \mathrm{~b}$ & \\
\hline ADM06 & Atención preventiva & 4182 & 54,1 & 4531 & 54,6 & 1,0 \\
\hline EAR11 & Infección del tracto respiratorio superior & 2622 & 33,9 & 3022 & 36,4 & 7,4 \\
\hline DEN01 & Trastornos bucales & 1734 & 22,4 & 2221 & 26,8 & $19,4^{c}$ \\
\hline ADM05 & Atención administrativa & 1326 & 17,2 & 1009 & 12,2 & $-29,1^{c}$ \\
\hline PSY06 & Problemas familiares y sociales & 863 & 11,2 & 953 & 11,5 & 2,9 \\
\hline PSY01 & Ansiedad, neurosis & 797 & 10,3 & 858 & 10,3 & 0,3 \\
\hline CAR14 & Hipertensión sin complicaciones graves & 726 & 9,4 & 754 & 9,1 & $-3,2$ \\
\hline MUS14 & Lumbalgia & 711 & 9,2 & 790 & 9,5 & 3,5 \\
\hline MUS01 & Signos y síntomas músculo-esqueléticos & 707 & 9,1 & 739 & 8,9 & $-2,6$ \\
\hline GAS07 & Gastroenteritis & 632 & 8,2 & 720 & 8,7 & 6,2 \\
\hline GSI01 & Signos y síntomas no específicos & 513 & 6,6 & 697 & 8,4 & $26,6^{c}$ \\
\hline CAR11 & Trastornos del metabolismo de los lípidos & 487 & 6,3 & 516 & 6,2 & $-1,3$ \\
\hline RES05 & Resfriado común & 464 & 6,0 & 510 & 6,1 & 2,4 \\
\hline EAR01 & Otitis media & 436 & 5,6 & 449 & 5,4 & $-4,0$ \\
\hline SKN01 & Contusiones y erosiones inespecíficas & 370 & 4,8 & 371 & 4,5 & $-6,6$ \\
\hline RES02 & Infección del tracto respiratorio inferior & 369 & 4,8 & 437 & 5,3 & $10,4^{\mathrm{c}}$ \\
\hline END06 & Diabetes tipo 2 & 350 & 4,5 & 373 & 4,5 & $-0,7$ \\
\hline SKN02 & Dermatitis y eczemas & 348 & 4,5 & 350 & 4,2 & $-6,3$ \\
\hline
\end{tabular}

a Según los conglomerados ampliados de diagnóstico ACG $^{\circledR}$ System (14).

b Porcentajes calculados con respecto al total de los episodios atendidos. A un paciente pueden corresponderle varios episodios. ${ }^{c}$ Diferencia significativa entre 2006 y $2007(P<0,001)$.

fueron en su conjunto $1,5 \%$ más complejos que los atendidos en 2006. El índice de eficiencia en el manejo de las visitas realizadas fue de 0,975 . En 2007 , los coeficientes de variación en la clasificación de los GCA fueron 73,4\% para los episodios y $115,2 \%$ para las visitas.

Según la matriz de correlaciones binarias y el modelo multifactorial (cuadro 4), hubo una elevada correlación lineal 
CUADRO 4. Poder explicativo (\% de la varianza) y correlaciones lineales $(r)$ de la clasificación de los grupos clínicos ajustados (GCA) ${ }^{a}$

\begin{tabular}{|c|c|c|c|}
\hline \multirow[b]{2}{*}{ Modelo estadístico } & \multicolumn{2}{|c|}{ Servicio de medicina } & \multirow[b]{2}{*}{ Total } \\
\hline & Familiar & Pediatría & \\
\hline \multicolumn{4}{|l|}{ Modelo de regresión lineal simple } \\
\hline \multicolumn{4}{|l|}{ Sin transformación logarítmica } \\
\hline Episodios & 79,6 & 54,0 & 74,5 \\
\hline Visitas & 42,2 & 42,3 & 42,0 \\
\hline \multicolumn{4}{|l|}{ Con transformación logarítmica (In) } \\
\hline Episodios & 79,5 & 57,6 & 74,8 \\
\hline Visitas & 54,5 & 51,2 & 53,4 \\
\hline \multicolumn{4}{|l|}{ Modelo de regresión lineal múltiple ${ }^{b}$} \\
\hline Variables de predicción: edad & 8,1 & 3,7 & 9,5 \\
\hline Variables de predicción: edad, sexo & 10,8 & 4,7 & 13,0 \\
\hline Variables de predicción: edad, sexo, episodios & 47,7 & 21,8 & 44,5 \\
\hline Matriz de correlaciones binarias (correlación lineal, $r$ ) & BUR $^{c}$ & Episodios & Visitas \\
\hline Episodios & 0,620 & & \\
\hline Visitas & 0,440 & 0,706 & \\
\hline Pesos relativos medio & & 0,629 & 0,485 \\
\hline
\end{tabular}

entre las visitas realizadas y los episodios atendidos $(r=0,706 ; P<0,001)$. Los episodios de atención explicaron una gran parte $(74,8 \%)$ de la varianza de la clasificación, mientras las visitas realizadas explicaron $53,4 \%$ de ella (después de una transformación logarítmica). Las varianzas explicadas en los modelos multifactoriales que utilizaron como variable dependiente los PRM fue de 9,5\% con la edad, $13,0 \%$ con la edad y el sexo, y $44,5 \%$ con la edad, el sexo y los episodios $(P<0,001)$.

La distribución del promedio de episodios y visitas según la edad de los pacientes atendidos durante el año 2007 se muestra en la figura 1. Los promedios de visitas más elevados se encontraron en los niños menores de 2 años y en los

FIGURA 1. Distribución del promedio de episodios y visitas según la edad de los pacientes atendidos, Municipio La Roca del Vallès, Barcelona, España, 2007

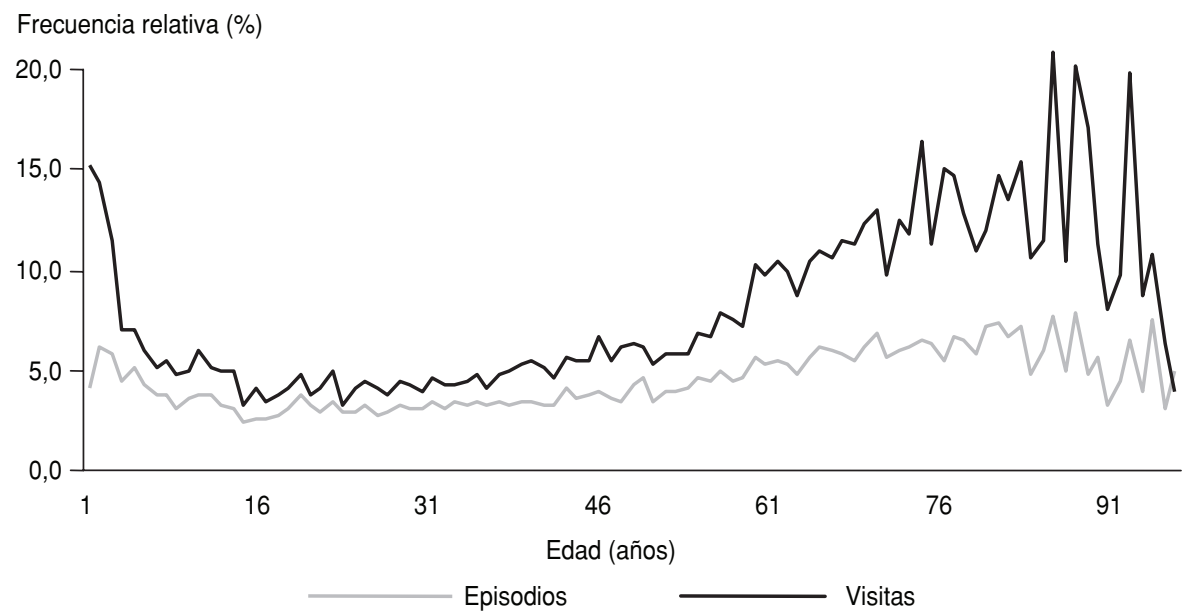

casa disponibilidad de información y de bases de datos amplias y confiables.

La organización de la APS en España, basada en la asignación de los equipos de salud a un territorio dado y una creciente informatización de sus centros, ofrece un marco idóneo para realizar este tipo de estudio de alcance poblacional a partir de la práctica clínica habitual. Es importante destacar que se requiere realizar una adecuada estandarización de las metodologías según las características de los pacientes y el número y la medida de las variables estudiadas para que los resultados obtenidos sean válidos, y aún así se deben interpretar dentro del escenario de la política sanitaria local y los proveedores de servicios y de gestión clínica existentes, por lo que se debe tener cautela al evaluar la validez externa de los resultados $(8-10,16,17)$.

En el presente estudio, la exhaustividad en el registro de la información general y los diagnósticos atendidos son dos aspectos fundamentales que se deben tener en cuenta para lograr una buena ubicación de cada paciente en el GCA que le corresponde. La estrategia debe basarse en que los profesionales del equipo de salud tengan una adecuada formación y mantengan una estrecha interrelación para mejorar el registro de la información y alcanzar un adecuado nivel de planificación y gestión clínicas (resultados en salud). A falta de una decisión política para elaborar un conjunto básico normalizado de datos para los servicios de APS o ambulatorios, se debe elevar el nivel de informatización de los centros y establecer mecanismos de consenso entre los profesionales para lograr una mayor uniformidad en los datos y la selección de los episodios. El reducido número de variables por paciente (edad, sexo y diagnóstico, no necesariamente correlativos en el tiempo) que se requiere para conformar los GCA se ajusta a las necesidades de la APS, donde se maneja un gran volumen de información diariamente, el tiempo asistencial es limitado, colaboran profesionales de diversos perfiles (médicos, enfermeros, trabajadores sociales y odontólogos, entre otros) y se repiten muchos pacientes en diferentes momentos $(16,18-21)$.

Los resultados de este estudio demuestran que la aplicación de la clasificación de los pacientes basada en los GCA permitió llegar a un grado de clasificación similar a los descritos en otros 
estudios, tanto españoles como extranjeros $(4,6,8,9-11)$, aunque se debe tener cautela al comparar los resultados alcanzados con versiones diferentes del agrupador. Además de mostrar cierto grado de inconstancia en cuanto a la complejidad en el conjunto de casos atendidos por los médicos de familia, el sistema de clasificación basado en los GCA permitió medir la atención sanitaria con un enfoque relativamente neutral. La agrupación de los episodios constituye un primer filtro que dificulta posibles cambios en la asignación de un paciente a un GCA específico, por lo que este tipo de error podría deberse más a aspectos organizativos y de asignación de pacientes que a diferencias en los estilos de la práctica clínica $(6,8-12)$.

El índice de riesgo de los casos estudiados fue de 1,015 y el índice de eficiencia en la gestión de las visitas fue de 0,975, lo que reflejó una mejoría en la gestión clínica del equipo de salud analizado con respecto al año anterior. No obstante, si existieran más centros que utilizaran los GCA y se pudieran comparar, se generarían datos más consistentes.

El índice de riesgo podría ayudar a ajustar la financiación y el posible establecimiento de tasas específicas de capitación, sin recurrir al cálculo complejo de los costos reales por paciente, ya que este sistema incorpora los PRM en cada categoría. En el orden práctico, resulta de interés la capacidad de este sistema para clasificar pacientes y distinguir las personas que realmente se han atendido de los usuarios potenciales. Así, la financiación de los equipos de APS podría basarse en: a) la ponderación de los costos estructurales vinculados con la accesibilidad, b) los costos variables según los casos atendidos (GCA) y la complejidad de las afecciones de los pacientes, y c) los objetivos de calidad derivados de la po- lítica deseada por el comprador y esperada por el cliente.

Por su parte, el índice de eficiencia en las visitas es un resultado positivo, pero incompleto, ya que solamente se valoró un aspecto de los costos directos. En un modelo de costos, las visitas realizadas pueden servir para imputar los costos de funcionamiento del equipo de salud (estimador de tiempo de los profesionales), pero para que el escenario sea más completo se deben cuantificar los costos variables de las pruebas diagnósticas y terapéuticas, las derivaciones a especialistas $\mathrm{y}$, sobre todo, las prescripciones farmacéuticas, ya que representan más de $50 \%$ del gasto sanitario en APS $(8,10,22)$. Esto generaría índices de eficiencia más completos.

En cuanto al poder explicativo de la clasificación según los GCA, los episodios registrados explicaron $74,8 \%$ de la varianza, mientras las visitas realizadas explicaron 53,4\%. Estos resultados son muy similares a los encontrados por otros autores $(6,8,10,22)$ y se deberán tener presente al analizar los costos de un equipo de salud. Cabe destacar que en los modelos multifactoriales, que utilizaron los PRM como variable dependiente, la edad mostró una varianza explicada de $9,5 \%$, mientras que con la edad y el sexo la varianza explicada fue de $13,0 \%$ y con la edad, el sexo y los episodios fue de $44,5 \%$. A pesar de que estos cálculos técnicos se pueden hacer por diversos métodos, con todos, al igual que con el empleado aquí, el poder explicativo de la clasificación aumenta al introducir variables relacionadas con los episodios, ya que reflejan el nivel de morbilidad (6, 8, 10, 12, 23-29).

La mayor limitación de este estudio es la baja validez externa de los resultados, tanto por el hecho de que el equipo estudiado no es representativo del universo general, como por el reducido número de pacientes analizados, lo que impide generalizar sus conclusiones. Otras limitaciones están relacionadas con el desarrollo de los sistemas de información utilizados en el estudio, posibles imprecisiones en la conversión de la CIAP a la CIE-9-MC e inexactitudes en el registro de las visitas y la gravedad de los episodios.

No obstante, este sistema de clasificación permitió agrupar de una forma relativamente sencilla y clínicamente coherente a los pacientes de una población a partir de su estado clínico. A pesar de las limitaciones planteadas, este sistema puede servir para ajustar los riesgos futuros (política sanitaria), medir el estado de salud (carga de morbilidad) de una población, cuantificar la eficiencia en el uso de los recursos y evaluar los resultados de los profesionales sanitarios (calidad asistencial). En el ámbito de la APS, este sistema de clasificación de pacientes puede ayudar a definir la financiación de los centros (pago capitativo) y administrar los recursos sanitarios (gestión clínica). Además, la poca capacidad de interacción clínica de los GCA, criticada por algunos profesionales sanitarios, puede verse contrastada con la información procedente de los CAD y los resultados en salud.

Agradecimientos. Se reconoce y agradece a B. Starfield, J. Weiner, K. Kinder y P. Boto, de la Universidad Johns Hopkins, por su apoyo en la realización del estudio, y a los profesionales sanitarios del centro de salud La Roca, sin cuya labor cotidiana no hubiera sido posible la realización de este estudio. Este trabajo recibió financiamiento parcial de PROSS, S.A., y el Fondo de Investigaciones Sanitarias de la Seguridad Social (PI 05/2837).

\section{REFERENCIAS}

1. Elola J, Daponte A, Navarro V. Health indicators and the organization of health care systems in Western Europe. Am J Public Health. 1995;85:1397-401.

2. Conesa A, Vilardell L, Casanellas JM, Torre P, Gelabert G, Trilla A, et al. Análisis y clasificación de las urgencias hospitalarias mediante los Ambulatory Patient Groups. Gac Sanit. 2003;17:447-52.

3. Fetter RG, Shin $Y$, Freeman JL, Averill RF, Thompson JD. Case mix definition by diagnosis-related groups. Med Care. 1980;18 (Suppl): 1-53.

4. Starfield B, Weiner J, Mumford L, Steinwachs D. Ambulatory care groups: a categorization of diagnoses for research and management. Health Serv Res. 1991;26:53-74.

5. Weiner JP, Starfield BH, Steinwachs DM, Mumford LM. Development and application of a population-oriented measure of ambulatory care case-mix. Med Care. 1991:29: $452-72$.
6. Meenan RT, Goodman MJ, Fishman PA, Hornbrook MC, O'Keeffe-Rosetti MC, Bachman DJ. Using risk-adjustment models to identify high-cost risks. Med Care. 2003;41: 1301-12.

7. Fusté J, Bolíbar B, Castillo A, Coderch J, Ruano I, Sicras A. Hacia la definición de un conjunto mínimo básico de datos de atención primaria. Aten Primaria. 2002;30:229-35.

8. Sicras-Mainar A, Serrat-Tarrés J, NavarroArtieda R, Llausí-Sellés R, Ruano-Ruano I, 
González-Ares JA. Adjusted clinical groups use as a measure of the referrals efficiency from primary care to specialized in Spain. Eur J Public Health. 2007;17:657-63.

9. Orueta JF, Urraca J, Berraondo I, Darpón J, Aurrekoetxea JJ. Adjusted clinical groups (ACGs) explain the utilization of primary care in Spain based on information registered in the medical records: a cross-sectional study. Health Policy. 2006;76:38-48.

10. Juncosa S, Bolíbar B. Descripció, comportament, usos i metodología d'utilització d'un sistema per mesurar la casuística en la nostra atenció primària: els ambulatory care groups. Barcelona: Fundació Salut, Empresa i Economia; 1999.

11. Bolaños-Carmona $V$, Ocaña-Riola R, PradosTorres A, Gutiérrez-Cuadra P. Variations in health services utilization by primary care patients. Health Serv Manage Res. 2002;15: $116-25$.

12. Juncosa S, Bolíbar B, Roset M, Tome R. Performance of an ambulatory case mix system in primary care in Spain: ambulatory care groups (ACGs). Eur J Public Health. 1999;9: $27-35$.

13. Lamberts $H$, Wood M, Hofmans-Okkes IM (eds). The International Classification of Primary Care in the European Community. With a multi-language layer. Oxford: Oxford University Press; 1993.

14. Johns Hopkins University. The Johns Hopkins $\mathrm{ACG}^{\circledast}$ Case-mix System version 7.0, April, 2005. Baltimore, MD: Johns Hopkins University; 2005. Hallado en http://www. acg.jhph. edu. Acceso el 13 de noviembre de 2009.
15. Expert Panel on Detection, Evaluation, and Treatment on High Blood Cholesterol in Adults. Third report of the National Cholesterol Education Program (NCEP) Expert Panel on Detection, Evaluation, and Treatment of High Blood Cholesterol in Adults. Adult Treatment Panel III final report. Bethesda, MA: National Institutes of Health 2002;106:3143-421.

16. Varela J, Berenguer J, Alonso E, Manzanera R, Picas JM. Los sistemas de información en atención primaria. Aten Primaria. 1988;5:543-7.

17. Sackett D, Rosenberg W, Gray J, Haynes R, Richardson WS. Evidence based medicine: what it is and what it isn't. Br Med J. 1996; 312:71-2.

18. Gosálbez-Pastor E, Pérez-Salinas I, DíezDomingo J, Ballester-Sanz A, Costa-Alcaraz MA. Sistemas de información en atención primaria: ¿debemos codificar con la CIE-9-MC? Aten Primaria. 2003;31:519-23.

19. Pedrera-Carbonell V, Gil-Guillén V, OrozcoBeltrán D, Prieto-Erades I, Schwarz-Chavarri G, Moya-García MI. Validez de la historia clínica y sistemas de información en los estudios de actividad asistencial en atención primaria. Aten Primaria. 2005;36:550-7.

20. Petersen LA, Pietz K, Woodard LD, Byrne M. Comparison of the predictive validity of diagnosis-based risk adjusters for clinical outcomes. Med Care. 2005;43:61-7.

21. Juncosa S, Bolíbar B, Roset M, Martínez C. Influencia de la unidad de análisis en los estudios de utilización de recursos en atención primaria. Gac Sanit. 1999;13:53-61.

22. Vargas I. La utilización del mecanismo de asignación per cápita: la experiencia de Cataluña. Cuad Gestión. 2002;8:167-78.
23. Sullivan CO, Omar RZ, Forrest CB, Majeed A Adjusting for case mix and social class in examining variation in home visits between practices. Fam Pract. 2004;21:355-63.

24. Starfield B, Lemke KW, Bernhardt T, Foldes SS, Forrest $\mathrm{CB}$, Weiner JP. Comorbidity: implications for the importance of primary care in 'case' management. Ann Fam Med. 2003;1: 8-14.

25. Carlsson L, Strender LE, Fridh G, Nilsson G. Types of morbidity and categories of patients in a Swedish county. Applying the Johns Hopkins Adjusted Clinical Groups System to encounter data in primary health care. Scand J Prim Health Care. 2004;22:174-9.

26. Reid RJ, MacWilliam L, Verhulst L, Roos N, Atkinson M. Performance of the ACG casemix system in two Canadian provinces. Med Care. 2001;39:86-99.

27. Wahls TL, Barnett MJ, Rosenthal GE. Predicting resource utilization in a veterans health administration primary care population: comparison of methods based on diagnoses and medications. Med Care. 2004;42:123-8.

28. Fishman PA, Goodman MJ, Hornbrook MC, Meenan RT, Bachman DJ, O'Keeffe Rosetti MC. Risk adjustment using automated ambulatory pharmacy data: the RxRisk model. Med Care. 2003;41:84-99.

29. Martínez C, Juncosa S, Roset M. ¿Está relacionada la gravedad con la utilización de recursos? Una exploración del Duke Severity of Illness Scale (DUSOI). Aten Primaria. 1998;22: 285-92.

Manuscrito recibido el 8 de diciembre de 2008. Aceptado para publicación, tras revisión, el 8 de abril de 2009.
ABSTRACT

\section{Adjusted clinical groups use
at a Spanish primary care \\ Adjusted clinical groups use
at a Spanish primary care center: a retrospective, population-based study}

Objectives. To evaluate the results of implementing a classification system based on adjusted clinical groups (ACG) at a primary health care (PHC) in a Spanish population. Methods. A retrospective, cross-sectional study based on the computerized medical records of outpatients seen in 2007 by the La Roca clinic, administered by a health services management company in La Roca del Vallès, Barcelona, Spain. The ACGs were formed according to the International Classification of Diseases, 9th Revision, Clinical Modification. The relative weight of each ACG's total average cost was calculated (in U.S. dollars) and based on these, the resources usage levels were established. The risk index (RI) and efficiency index (EI) for 2006 and the classification's explanatory power were determined.

Results. A total of 8294 patients were studied ( $82.7 \%$ coverage), with an average of 4.1 incidents per patient, 6.9 visits per patient, and 5.7 visits per person per year. Seven GCAs accounted for $51.0 \%$ of patients seen. The RI was 1.015, the EI was 0.975 visits, and the explanatory power of the ACG classification was $53.4 \%$ for visits and $74.8 \%$ for incidents.

Conclusions. The ACG system allowed this patient population to be grouped by clinical status, which can help to, among other things, allocate resources and evaluate PHC team efficiency.

Key words Diagnosis-related groups; primary health care; efficiency, organizational; Spain. 